\title{
Improved Acquisition Method of GPS Signal by Wavelet De-noising
}

\author{
Chenlan WANG \\ School of Communication \& Information Engineering, University of Electronic Science and Technology of \\ China, Chengdu, China
}

Zhong WANG

School of Electrical Engineering and Information, Sichuan University, Chengdu, China

\begin{abstract}
To solve the problem that it is difficult to capture the weak GPS signal, an improved wavelet noise reduction algorithm is proposed. By integrating correlation integral differential accumulation method, wavelet decomposition and signal reconstruction, the algorithm can improve the signal-to-noise ratio effectively but not increase the computing time notably. In order to obtain a higher signal-to-noise ratio, a new self-transformation threshold choosing method is introduced in this paper. It is optimizing the coefficients of wavelet decomposition, based on the different behavior of the noise in different decomposition level. The simulation shows that the de-noising effects are improved obviously, the wavelet de-noising method is better than the traditional method.
\end{abstract}

KEYWORD: self-transformation threshold; wavelet de-noising; weak GPS signal acquisition component

\section{INTRODUCTION}

With the progress of technology, navigation technology has been more widely used. Traditional GPS receiver can be very convenient for highprecision three-dimensional positioning. However, under the conditions of the complexity of the interior, forest, tall over the city center positioning, The GPS signal was severe attenuation. Signal power is much lower than traditional GPS receiver. Common receiver cannot correctly capture and track to signal. This will greatly limit the application of the GPS receiver. Navigation technology under weak signal conditions, whether in military or civilian areas have a greater significance. At present, research on acquisition of weak GPS signal is relatively less. The literature [1] has proposed a double block zero extension based on (double block zero padding, DBZP) differential correlation acquisition algorithm. The literature [2] improves the ability to capture weak signals by extending time to calculate coherent and incoherent integral. The current method is capable of weak GPS capture to some extent, but when the carrier to noise ratio is low to the above method is not enough to capture the signal. This paper presents a method of improved wavelet algorithm with adaptive threshold improvement combined, and through the MATLAB simulation results show that the method can reliably working under low SNR condition. Compared with the traditional method, this method can not only improve the capture signal-to-noise ratio, but not significantly increase the capture time.

\section{WAVELET DE-NOISING PRINCIPLE}

Wavelet analysis is emerging in recent years a signal processing method. The advantage is in the time domain and frequency domain also has good local properties. The basic idea is the signal at different scales of decomposition, and analysis of the corresponding characteristics of high frequency coefficients. Finally, Noise system was threshold processed to achieve the noise reduction process [3]. Experiments confirmed that wavelet de-noising can improve the SNR to a certain extent. This has been widely used in signal detection, image processing, information fusion, etc, which achieved good results [4]. The application of wavelet de-noising in the field of satellite navigation has become a hot spot. Using the wavelet decomposition of the onedimensional signal noise reduction processing is one of the important applications of the wavelet decomposition. Following a brief description for the principle of wavelet de-noising is given.

One-dimensional model of the noisy signal can be expressed as

$$
s(i)=f(i)+e(i), \quad i=0,1, ? \quad n-1
$$


Among this, $f(i)$ is the real signal. $e(i)$ is noise. $s(i)$ is the noisy signal. Signal after the wavelet decomposition will show different characteristics. The useful signal is usually reflected in the low-frequency part, and the noise signal is often high-frequency signals [5]. Signal after the $j$ layer wavelet decomposition can be expressed as

$$
D W(z)_{j}=A_{j}+\sum_{i=1}^{j} D_{j}
$$

$A_{j}$ shows low-frequency characteristics, named approximation, which characterized mainly part of the useful signal. $D_{j}$ shows high-frequency characteristics, called detail, which represents the white noise.

The basic idea of wavelet de-noising is to transform the signals of different scales, decompose them into the $\mathrm{J}$ layer, and then analyses the high frequency coefficients [6]. According to the noise decomposition layer leading scale, by threshold processing function and proper threshold, the high frequency coefficients of wavelet coefficient threshold processing is new, and then use the new wavelet coefficients to reconstruct the signal processing, so as to achieve de-noising purpose. [7]:

De-noising processing is divided into three steps

First is wavelet decomposition of the onedimensional signal, choosing a wavelet basis and determining the decomposition level $\mathrm{N}$. The signals would be decomposed to $\mathrm{N}$ layers.

Second is threshold quantization on highfrequency coefficients of wavelet decomposition. The appropriate threshold function will be selected. High-frequency coefficients of each layer from layer 1 to layer $\mathrm{N}$ will be processed by a suitable threshold.

Third is reconstruction of one-dimensional wavelet. One-dimensional signal will be reconstructed based on the wavelet coefficients from the threshold quantization.

\section{IMPROVED ACQUISITION ALGORITHM}

\subsection{Integral differential accumulation [8]}

Define abbreviations and acronyms the first time they are used in the text, even after they have been defined in the abstract. Abbreviations such as IEEE, SI, MKS, CGS, sc, dc, and rms do not have to be defined. Do not use abbreviations in the title or heads unless they are unavoidable.

GPS IF signal multiplied with the local PRN code and carrier. After correlation integral, in-phase and quartered components can be expressed as

$$
\begin{aligned}
& I=\frac{\sin \left(\pi_{\Delta} f T\right)}{\left(\pi_{\Delta} f T\right)} \sqrt{2 \frac{S}{N} T} R(\tau) D \cos (\Delta \varphi)+I \\
& Q_{i}=\frac{\sin \left(\pi_{\Delta} f_{i} T\right)}{\left(\pi_{\Delta} f_{i} T\right)} \sqrt{2 \frac{S}{N_{0}}} T R\left(\tau_{i}\right) D_{i} \sin \left(\Delta \varphi_{i}\right)+Q_{n i}
\end{aligned}
$$

Where i represents the i-coherent accumulator, $D_{i}$ is Navigation message data corresponding to the accumulated value (value of \pm 1 ), $\Delta \varphi$ said the initial phase, $\Delta f$ said frequency difference is the cumulative time. $R\left(\tau_{i}\right)$ is correlation function for the $\mathrm{C} / \mathrm{A}$ code since. $N_{o}$ is bilateral baseband noise power spectral density. $I_{n i}, Q_{n i}$ said the noise inphase and quartered components.

Removing the amplitude factor, the autocorrelation function coefficients can be expressed as vector form

$$
D_{i} e^{j\left(2 \pi \Delta f_{i} T_{i}+\Delta \varphi\right)}
$$

Followed by $\mathrm{i}+1$ vector is

$$
D_{i+1} e^{j\left(2 \pi \Delta f_{i} T_{i+1}+\Delta \varphi\right)}
$$

Differential accumulation process is

First, formula (5)'s conjugate multiplied by formula (6), the result is $D_{i} D_{i+1} e^{j 2 \pi \Delta f_{i} T} \cdot e^{j 2 \pi \Delta f_{i} T}$ is fixed value.

Secondly, the results will be accumulated.

Finally, the sum of the modulus square was demand as the test statistic.

\subsection{Wavelet acquisition algorithm}

Improved wavelet acquisition algorithm flow is shown in Figure 1

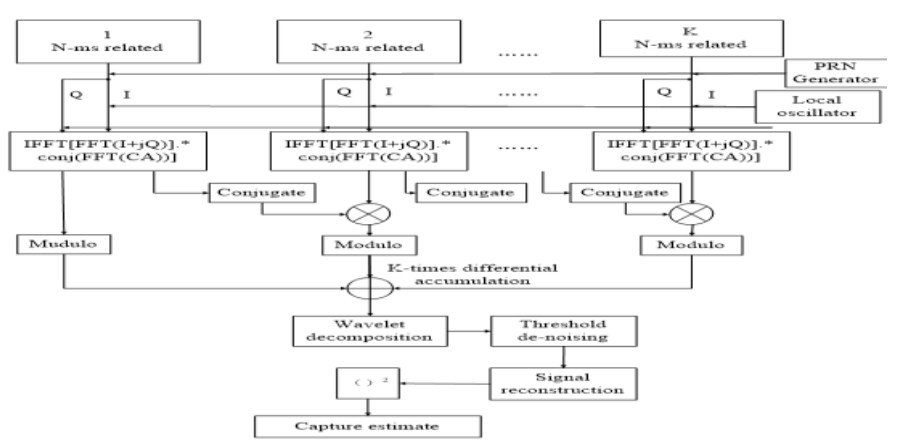

Figure 1. Improved wavelet de-noising acquisition algorithm

Firstly, The IF signal would be processed by N-ms coherent integration, outputting coherent matrix $\mathrm{R}$ (k). R (k) Conjugated and multiplied the adjacent coherent matrix R (k-1), getting the matrix $\mathrm{Y}(\mathrm{k})$. By using the K-times differential accumulation method, $\mathrm{Y}(\mathrm{k})$ could generate the matrix $Z(K)$. Then each row vector $Z(K)$ in $Z(K)$ as the input of the Wavelet Transform, one-dimensional wavelet 
decomposition, threshold noise reduction and signal reconstruction is done. The individual rows of data arranged are reconstructed of by row order to get the reconstruction matrix $\hat{Z}(K)$. Then the matrix $\hat{Z}(K)$ is squared, to further improve the signal-to-noise ratio. Finally, the squared matrix peak and capture the gate limits compared to the results are obtained capture.

Since the original GPS signal using C / A code modulation, the signal performances characteristics of strong white noise. This can not be used as the input of the wavelet filter. After coherent integration, non-coherent signal accumulation, the useful signal is no longer showing the white noise characteristics, but at the same time, the noise characteristics does not conform to the Gaussian distribution, showing the center of the chi-square distribution [9]. After several experiments, using the differential accumulation alternative non-coherent accumulation, noise characteristics is similar with the Gaussian distribution [10], when the cumulative number is large. Therefore, before carrying out the wavelet threshold de-noising, first the correlation matrix is differential accumulated.

\section{ADAPTIVE THRESHOLD}

Wavelet decomposition of the correlation integral, differential accumulation of data, it can be found in noise are reflected at all scales, and has its own mean and variance. If we use the traditional unified threshold de-noising method, it may not be able to filter out the vast majority of noise. Therefore, in order to achieve higher signal to noise ratio, threshold selection method needs to make improvements. If the layer $j$ of the wavelet detail coefficients is $d_{j k}\left(k=1,2 \ldots \ldots L_{j}, L_{j}\right.$ is the length of the wavelet coefficients of layer $j$ ). Standard deviation $\operatorname{std}\left(d_{j k}\right)$ can be regarded as the reference value of the noise of the layer. So the threshold of the $\mathrm{j}$ layer is chosen as

$$
\lambda_{j}=\mathrm{A}_{j} * \operatorname{std}\left(d_{j k}\right) / 0.6745
$$

$1 / 0.6745$ is experience, $A_{j}$ is linear reduction factor, the value is

$$
\mathrm{A}_{j}=1.1-0.05 j
$$

This form of linear reduction factor is used, because noise energy is concentrated in a smaller number of layers decomposed layer. Its wavelet coefficients rapidly decay with the increase of decomposition level. The useful signal has embodied at each layer.

\section{EXPERIMENT}

DB2 is chosen as the wavelet basis function. The decomposition level is set to 8 layers. The soft threshold function is used. Simulated signals are used as source signal. Sampling frequency is $5 \mathrm{MHz}$, $\mathrm{IF}=1.25 \mathrm{MHz}$.

When $C / N_{o}=32 \mathrm{~dB}-\mathrm{Hz}$, the results of differential coherent acquisition method and wavelet acquisition method were shown in Figure 2 and Figure 3.

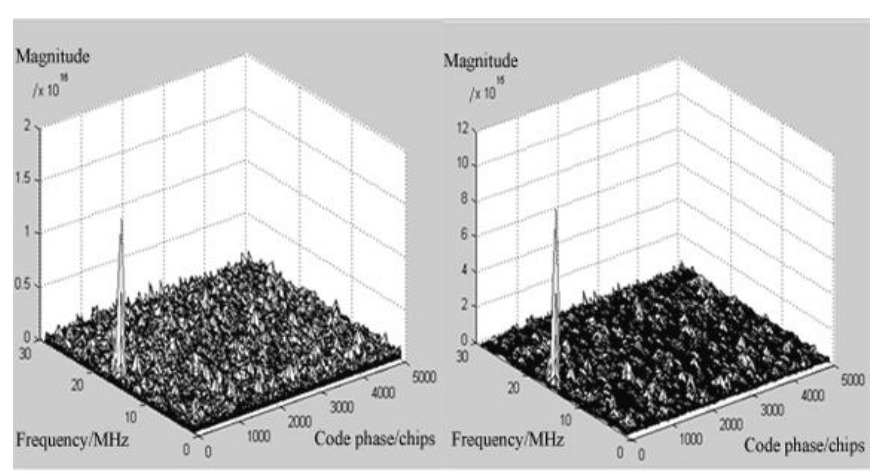

(a) 32dB-Hz Differential acquisition

(b) 32dB-Hz Wavelet acquesition

Figure 2. 32dB-Hz differential acquisition and wavelet acquisition results of $3 \mathrm{D}$

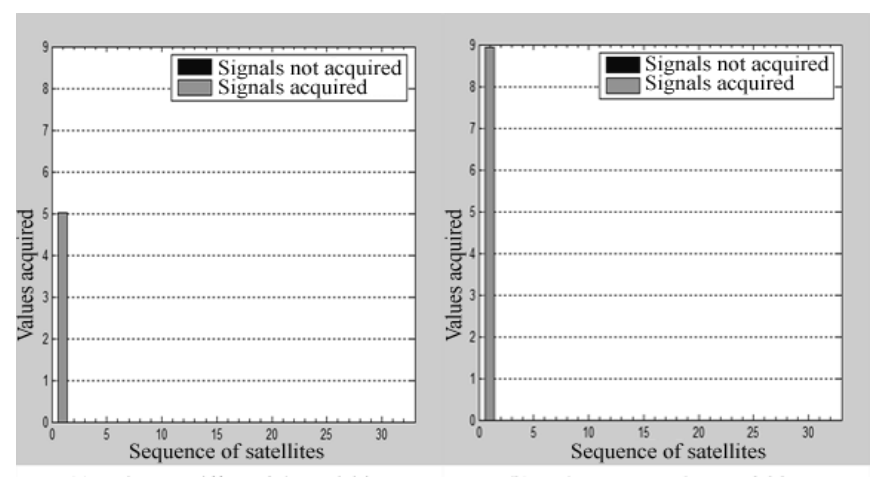

(a) $32 \mathrm{~dB}-\mathrm{Hz}$ Differential acquisition

(b) $32 \mathrm{~dB}-\mathrm{Hz}$ Wavelet acquisition

Figure 3. 32dB-Hz differential acquisition and wavelet acquisition results of $2 \mathrm{D}$

From figure 2 we can find that the noise content is smaller using wavelet acquisition. In figure 3, the SNR is bigger using wavelet acquisition.

When $C / N_{o}=29 \mathrm{~dB}-\mathrm{Hz}$, the results of differential coherent acquisition method and wavelet acquisition method also were better.

Using only the differential method cannot successfully capture the signal, but the wavelet method can do it. So, it is better than traditional method.

The results using traditional threshold selection method and adaptive threshold selection method is shown in Figure 4 and Figure 5. 


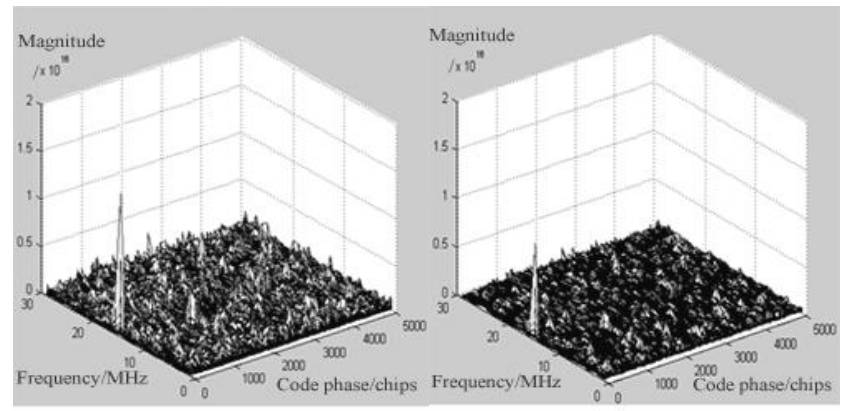

(a) $\lambda=\sigma * \operatorname{sqrt}(\ln (\mathrm{N}))$

(b) $\lambda_{\mathrm{j}}=\mathrm{Aj} * \operatorname{std}\left(\mathrm{d}_{\mathrm{jk}}\right) / 0.6745$

Figure 4. different threshold selection methods 3D

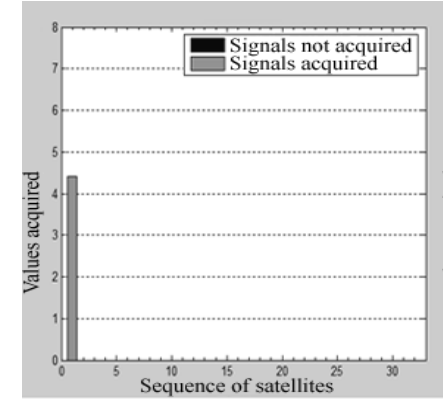

(a) $\lambda=\sigma * \operatorname{sqrt}(\ln (\mathrm{N}))$

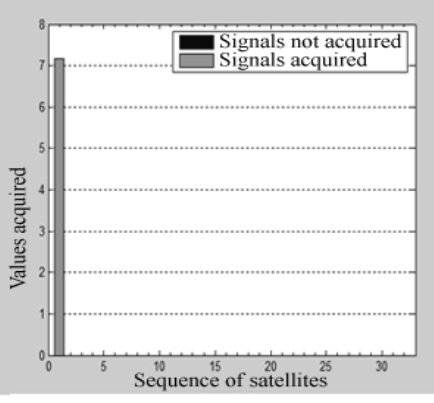

(b) $\lambda_{j}=A j * \operatorname{std}\left(d_{j k}\right) / 0.6745$
Figure 5. Different threshold selection methods acquisition value

From these figures, it is not difficult to see that, improved adaptive threshold selection method of the residual noise is smaller than that of the traditional uniform threshold setting method, and the capture value is far greater than the traditional uniform threshold setting method.

\section{CONCLUSION}

The method used in this paper improves the traditional method by using wavelet de-noising. It mainly comprises the following steps: firstly, the Ktimes difference accumulation coherent integration, so that the noise characteristics in line with the Gauss distribution, suitable as the input of wavelet transform. Then the correlation matrix accumulation after wavelet decomposition, wavelet threshold denoising processing, finally through wavelet reconstruction to restore the signal is pure, in the capture of square after the final decision. Verified by MATLAB wavelet capture method can effective signal-to-noise improvement to a certain extent than, but not significantly increase the computation time, and the traditional differential capture method has better performance compared to capture. Improved wavelet algorithm can achieve the signal condition of weak signal acquisition, and the improved threshold setting method is superior to the traditional threshold setting method.

\section{ACKNOWLEDGMENT}

This work funded by the national aeronautics science foundation of china (No: 20100119004), and i would like to thank the many people who made this design a success, zhong wang, yangyi zhang and many more.

\section{REFERENCES}

[1] Mo Jianwen. "GPS signal acquisition algorithm based on DBZP differential coherent," Systems Engineering and Electronics, vol.34, no.6, pp.1085-1089, 2012.

[2] $\mathrm{Hu}$ Congwei,Li Xiaoling,An Lei, “A improved acquisition method of weak GPS signal," Geomatics and Information Science of Wuhan University, vol.33,no.8,pp.821-823, 2008.

[3] Robert J. Mcaulay, Marilyn L. Malpass, “ Speech enhancement using a soft-decision noise suppression filter," IEEE Transaction on Acoustics, Speech and Signal Processing, vol.16, no.9,pp. 328-335, 1980.

[4] Wang Fei,"Study on Application of Combination of Wavelet and Fractal in Image Processing, " Science \& Technology Vision, no.28,pp.268-269, 2012.

[5] Mallat S, Zhong S. "Characterization of signals from multiscale edges," IEEE Trans. On Pattern Analysis and Machine Intelligence, vol.14,no.7,pp.710-732. 1992.

[6] LIU Yang,JIN Tian, "Application of wavelet de-noising in weak satellite signal acquisition,"Systems Engineering and Electronics, vol.33, No.4, pp.:842-846, 2011

[7] Donoho D L, Johnstone J M. "Ideal spatial adaption by wavelet shrinkage," Biometrika,vol. 81, no.3, pp. 425455,1994.

[8] PI Yiming,ZHANG Jing,CAI Changting. "Research of Differential Correlation Acquisition Algorithm in GPS,"GNSS World of China ,No.4, 2006

[9] Steven M K. "Fundamentals of statistical signal processing-estimation and detection theory,"Luo P F, Zhang W M, Liu Z, et al,trans. Beijing:House of Electronics Industrypp.470-499, 2006.

[10]Elders-Boll H, Dettmar U. "Efficient differentially coherent code/ Doppler acquisition of weak GPS signals, spread spectrum techniques and applications," Proc. of IEEE Eight International Symposium on Spread Spectrum Techniques and Applications, pp.731- 735, 2004. 